\title{
Microarray analysis reveals an important role for dietary L-arginine in regulating global gene expression in porcine placentae during early gestation
}

\author{
Xilong Li ${ }^{1, \S}$, Gregory A. Johnson ${ }^{2}$, Huaijun Zhou ${ }^{3, \S}$, Robert C. Burghardt ${ }^{2}$, Fuller W. Bazer ${ }^{1}$, \\ Guoyao $\mathrm{Wu}^{1, *}$ \\ ${ }^{1}$ Departments of Animal Science, Texas A\&M University, College Station, TX 77843, USA \\ ${ }^{2}$ Departments of Veterinary Integrative Biosciences, Texas A\&M University, College Station, TX 77843, USA \\ ${ }^{3}$ Departments of Poultry Science, Texas A\&M University, College Station, TX 77843, USA \\ *Correspondence: g-wu@tamu.edu (Guoyao Wu) \\ $\S$ The current address of Xilong Li is Institute of Feed Research, The Chinese Academy of Agricultural Sciences, 100081 Beijing, China. \\ The current address of Huaijun Zhou is Department of Animal Science, University of California at Davis, Davis, CA 95616, USA. \\ Academic Editor: Graham Pawelec \\ Submitted: 22 November 2021 Revised: 28 December 2021 Accepted: 4 January 2022 Published: 18 January 2022
}

\begin{abstract}
Background: Increasing the dietary provision of L-arginine to pregnant swine beginning at Day 14 of gestation enhances embryonic survival, but the underlying mechanisms are largely unknown. Objective: This study determined the effects of dietary supplementation with $0.8 \%$ L-arginine to gilts between Days 14 and 25 of gestation on the global expression of genes in their placentae. Methods: Between Days 14 and 24 of gestation, gilts were fed $2 \mathrm{~kg}$ of a corn- and soybean meal-based diet (containing $12.0 \%$ crude protein and $0.70 \% \mathrm{Arg}$ ) supplemented with $0.8 \% \mathrm{~L}$-arginine or without L-arginine $(0.0 \%$; with $1.64 \% \mathrm{~L}$-alanine as the isonitrogenous control). On Day 25 of gestation, $30 \mathrm{~min}$ after the consumption of their top dressing containing $8 \mathrm{~g} \mathrm{~L}$-arginine or $16.4 \mathrm{~g} \mathrm{~L}$-alanine, gilts underwent hysterectomy to obtain placentae, which were snap-frozen in liquid nitrogen. Total RNAs were extracted from the frozen tissues and used for microarray analysis based on the 44-K Agilent porcine gene platform. Results: L-Arginine supplementation affected placental expression of 575 genes, with 146 genes being up-regulated and 429 genes being down-regulated. These differentially expressed genes play important roles in nutrient metabolism, polyamine production, protein synthesis, proteolysis, angiogenesis, immune development, anti-oxidative responses, and adhesion force between the chorioallantoic membrane and the endometrial epithelium, as well as functions of insulin, transforming growth factor beta, and Notch signaling pathways. Conclusion: Dietary supplementation with L-arginine plays an important role in regulating placental gene expression in gilts. Our findings help to elucidate mechanisms responsible for the beneficial effect of L-arginine in improving placental growth and embryonic/fetal survival in swine.
\end{abstract}

Keywords: Amino acids; Metabolism; Nutrition; Pigs; Placenta; Pregnancy

\section{Introduction}

There is growing interest in the nutritional role of Larginine (Arg) to enhance litter size in livestock species [13]. However, only a few studies have been conducted to explore the underlying mechanisms [4-6]. Thus, there is a limited understanding of regulatory functions of Arg in the placenta. Results of recent studies indicated that Arg is not only a building block for proteins, but also has multiple physiological roles in cell signaling and function [7,8]. For example, Arg stimulates the production of nitric oxide (NO) and polyamines (key regulators of cell growth and development) by placental cells $[9,10]$, as well as the placental expression of aquaporins and the transport of water across the placentae [6]. In addition, Arg may influence the expression of genes related to amino acid transport, anti-oxidative responses, and protein synthesis in mammalian cells [9,11]. As an approach to understanding how Arg acts on the placentae at the gene level, we used the 44-K Agilent porcine gene platform to determine changes in global gene expres- sion in placentae at Day 25 of gestation from gilts receiving dietary Arg supplementation between Days 14 and 25 of gestation. This nutritional method is effective in enhancing placental growth and embryonic survival in swine [12].

\section{Materials and methods}

\subsection{Animals and diets}

The experimental design, including the diets of gilts before and after breeding, has been described by Li et al. [12]. Briefly, gilts (F1 crosses of Yorkshire $\times$ Landrace sows and Duroc $\times$ Hampshire boars) were checked daily for estrus with boars and bred $12 \mathrm{~h}$ and $24 \mathrm{~h}$ after the onset of the second estrus detected by the boars. Immediately after breeding, gilts were assigned randomly to one of the two treatment groups [0.0\% Arg (with $1.64 \%$ L-alanine as the isonitrogenous control) or $0.8 \% \mathrm{Arg}]$. There were 10 gilts (individually penned) per treatment group. Between Days 14 and 23 of gestation, gilts were fed twice daily (07:00 h 
and 18:00 h) $1 \mathrm{~kg}$ of a corn- and soybean meal-based diet (containing $12.0 \%$ crude protein and $0.70 \%$ Arg) supplemented with $0.0 \%$ Arg (1.64\% L-alanine; Control group) or $0.8 \%$ Arg (Arg group) [12]. The total feed intake of each gilt was $2 \mathrm{~kg}$ per day. On each day, L-alanine or Arg was mixed with cornstarch and then added to the basal diet as a top dressing consumed by each gilt. On Day 24 of gestation, gilts were fed once $(08: 00 \mathrm{~h})$ with $2 \mathrm{~kg}$ of diet supplemented with either $0.8 \%$ Arg or $1.64 \%$ Ala. On Day 25 of gestation, $22 \mathrm{~h}$ after the last meal and $30 \mathrm{~min}$ after the consumption of their top dressing containing $8 \mathrm{~g}$ Arg or $16.4 \mathrm{~g} \mathrm{L-alanine,} \mathrm{gilts} \mathrm{were} \mathrm{prepared} \mathrm{for} \mathrm{surgery}$ and hysterectomized to obtain uteri and conceptuses (fetus and placenta). L-Alanine, rather than a mixture of amino acids, was used as the isonitrogenous control, because it is rapidly catabolized by pigs $[5,12]$, is not a substrate for arginine synthesis $[5,12]$, and does not affect any of the measured variables of reproductive performance on Day 25 of gestation (the number of corpora lutea; uterine, placental, and embryonic/fetal weights; the total number of fetuses, embryonic survival, and the number of live fetuses; and volumes of amniotic and allantoic fluids, compared with non-supplemented gilts $[2,3,5,6,12])$. This research was approved by Texas A\&M University Animal Use and Care Committee.

\subsection{Collection of placentae}

Each placenta was obtained from a live fetus. A portion of the placenta was immediately snap-frozen in liquid nitrogen. All snap-frozen samples were stored at $-80{ }^{\circ} \mathrm{C}$ until analyzed. Eight gilts (three placentae from each gilt) in each group were selected randomly for the extraction of total RNA.

\subsection{Total RNA isolation}

Total RNA was isolated from the frozen placenta (approximately $30 \mathrm{mg}$ ) according to the manual of the RNeasy Mini Kit (Qiagen Inc., Valencia, CA) [4]. The quantity of the total RNA was measured by NanoDrop 1000 Spectrophotometer (Thermo Scientific, USA). The quality of total RNA was determined by $1 \%$ agarose electrophoresis. In addition, we determined the ratio of absorbance at 260 $\mathrm{nm}$ and $280 \mathrm{~nm}$, which was used to assess the purity of RNA, was approximately 2.0 for the total RNA isolated from porcine placentae. The total RNA from 3 placentae from each gilt was combined at equal quantity to represent one biological replicate, and there were 8 biological replicates for each treatment group in the following microarray analysis.

\subsection{Microarray analysis}

Total RNA (400 ng) was reverse-transcribed to cDNA. T7 RNA polymerase-driven RNA synthesis was used for the preparation and labeling of cRNA with Cy3 or Cy 5 dye. In each treatment group, 4 samples were treated with the
Cy3 (green) dye, and 4 samples were treated with the Cy5 (red) dye. The labeled cRNA probes were purified with the RNeasy Mini Kit (Qiagen Inc., Valencia, CA). Purified cRNA was quantified with the NanoDrop 1000, and $825 \mathrm{ng}$ of each was hybridized on the 44-K Agilent porcine gene expression microarray (Agilent, Santa Clara, CA). This array included 43,803 probes that were prepared using gene sources from RefSeq, UniGene, and TIGR. The slide format was printed using the Agilent's 60-mer SurePrint technology. The hybridized slides were washed according to the manual of a commercial kit (Agilent Technology, Palo Alto, CA), followed by scanning with a Genepix 4100A scanner (Molecular Devices Corporation, Sunnyvale, CA) with the tolerance of saturation setting of $0.005 \%$. A locally weighted linear regression (LOWESS) method was applied to normalize the data by the median of the signal intensity and local background values. SAS 9.1.3 program (SAS Institute Inc. Cary, NC) for the mixed model was used to analyze the normalized data [13]. Statistical significance to detect differentially expressed genes was determined by the approximate $t$-test for least-square means, where $p<0.05$ was considered to be statistically significant. The false discovery rate ( $\mathrm{Q}$ value) was calculated for each $p$-value using the $\mathrm{R}$ program [13]. Genes were annotated by the basic local alignment search tool (BLAST) in the database of the National Center for Biotechnology Information (NCBI) and the Institute for Genomic Research (TIGR). The database for annotation, visualization, and integrated discovery (DAVID) version 6.7 was used to generate specific functional annotations of biological processes for the differentially expressed genes [14].

\subsection{Interaction pathways analysis for selected genes that were differentially expressed in the placentae of Arg-supplemented gilts}

GO terms for biological processes (GO_TERM_BP) and KEGG pathways were identified for differentially expressed genes (both up- and down-regulated genes) using the database for DAVID version $6.8[15,16]$. Ensembl gene IDs were converted to official gene symbols for input into DAVID using Ensembl's Biomart, which is an opensource software and data service to the international scientific community (https://m.ensembl.org/info/data/biomar t/index.html). Significance cutoff was $p<0.05$.

\subsection{Quantitative real-time PCR}

Total RNA $(1 \mu \mathrm{g})$ from each sample was used for cDNA synthesis with a random hexamer primer of a Thermoscript RT-PCR system kit (Invitrogen, Carlsbad, CA) according to the manufacturer's instructions. The cDNAs were quantified by quantitative RT-PCR using the ABI Prism 7900HT system with SYBR Green PCR Master Mix (Applied Biosystems, Foster, CA) [4]. The primers for each gene were designed by using the Oligo6 program (www.oligo.net; Table 1). The cycling conditions of quan- 
Table 1. Sequence and optimal annealing temperatures for primers used in quantitative RT-PCR analyses.

\begin{tabular}{|c|c|c|c|c|}
\hline Accession No. & Gene & Primer sequence & Product length (bp) & Annealing Temp. $\left({ }^{\circ} \mathrm{C}\right)$ \\
\hline NM_001001861 & CXCL2 & $\begin{array}{l}\text { Forward: 5'- CACTGTGACCAAACGGAA -3' } \\
\text { Reverse:5'- GTTGGCACTGCTCTTGTTT-3' }\end{array}$ & 120 & 53 \\
\hline NM_214003 & $I G F B P 2$ & $\begin{array}{c}\text { Forward: 5'- GTGGATGGGAACGTGAACTT-3' } \\
\text { Reverse:5'- GTGCTGCTCCGTGACTTTCT-3' }\end{array}$ & 111 & 56.8 \\
\hline TC267605 & PFKFB1 & $\begin{array}{c}\text { Forward: 5'- GCCTAAGATGACTCAAGAGA-3' } \\
\text { Reverse:5'- CGTGGAGATGTAGGTCTTT-3' }\end{array}$ & 187 & 53.3 \\
\hline NM_213963 & PPARGC & $\begin{array}{l}\text { Forward: 5'- AACCCACAGAGACCCGAAAC-3' } \\
\text { Reverse:5'- AAATGTTGCGACTGCGATTG-3' }\end{array}$ & 82 & 53 \\
\hline AK231515 & Presenilin 2 & $\begin{array}{l}\text { Forward: 5'- AAGGAGCACAGCGGACTCT-3' } \\
\text { Reverse:5'- TGGGTACTGAACGGGTGTTT-3' }\end{array}$ & 299 & 57 \\
\hline TC275071 & $R A G-2$ & $\begin{array}{l}\text { Forward: 5'- ATGCCAGATCCTTAACCCAC-3' } \\
\text { Reverse:5'- GCAGCAGAAATGAATCCAAC-3' }\end{array}$ & 82 & 53 \\
\hline BI341657 & RasGEF & $\begin{array}{l}\text { Forward: 5'- CTCCCATCTACAGCGAGGAA-3' } \\
\text { Reverse: 5'- GAGCGTGGTCCTGAGGGTCT-3' }\end{array}$ & 104 & 56 \\
\hline TC243513 & $R H B G$ & $\begin{array}{l}\text { Forward: 5'- GTGCCTACTTTGGGTTGGTC-3' } \\
\text { Reverse:5'- ATGGCAAAGAGGTCCGAATG-3' }\end{array}$ & 103 & 56 \\
\hline TC257543 & $R U 2 S$ & $\begin{array}{l}\text { Forward: 5'- CACTTCTGGAACCCTGCACT-3' } \\
\text { Reverse:5'- TGATCCCACTGATTCAAGGC-3' }\end{array}$ & 103 & 53 \\
\hline NM_001001863 & TNNT3 & $\begin{array}{l}\text { Forward: 5'- CCTGTACCARCTGGAGATTG-3' } \\
\text { Reverse: 5'- CTGAGGTTGATGATGTCGTA-3' }\end{array}$ & 78 & 51 \\
\hline DQ225365 & Tubulin $\alpha$ & $\begin{array}{l}\text { Forward: 5'-GCAGTGTTTGTAGACCTG GA-3' } \\
\text { Reverse:5'-CAATGGTGTAGTGACCTCGG-3' }\end{array}$ & 139 & 55 \\
\hline EU288086 & MTOR & $\begin{array}{l}\text { Forward: 5'- GTCTCTATCAAGTTGCTGGC-3' } \\
\text { Reverse: 5'- CTTTCGAGATGGCAATGGAA-3' }\end{array}$ & 126 & 53 \\
\hline NM_001012613 & $S L C 7 A 1$ & $\begin{array}{c}\text { Forward: 5'- ACTCGACTCTCGTGGACCTT-3' } \\
\text { Reverse:5' GGTCAGTTGACTTTCTGCCT-3' }\end{array}$ & 134 & 54 \\
\hline
\end{tabular}

Primers were prepared using the oligo6 program (www.oligo.net).

titative RT-PCR amplification were: 1 cycle at $95{ }^{\circ} \mathrm{C}$ for 10 min, 40 cycles at $95^{\circ} \mathrm{C}$ for $15 \mathrm{~s}$ and optimal annealing temperature for $1 \mathrm{~min}$. The porcine tubulin $\alpha$ gene was used as the housekeeping gene, and its expression was not affected by dietary Arg supplementation. Dissociation curves were performed at the end of amplification for validating data quality. For the RT-PCR analysis, slope values ranged from -3.51 and -3.32 , which corresponded to the reaction efficiencies of $93 \%$ and $100 \%$, respectively.

All samples were run in triplicate and the average critical threshold cycle $(\mathrm{Ct})$ was used to calculate the relative mRNA levels of target genes by the $2^{-\Delta \Delta C T}$ method [17]. We chose four significantly increased genes with a fold change more than 1.5 , four significantly decreased genes with a fold change more than 2 , and two genes with no change in mRNA levels based on microarray analysis to run quantitative real-time PCR for verifying the microarray data.

\subsection{Statistical analysis}

Data were analyzed by the unpaired $t$-test using the SPSS (Version 15.0, Chicago, IL). Gilt was considered as the experimental unit. Probability values $<0.05$ were considered statistically significant.

\section{Results}

3.1 Global change in placental mRNA levels based on microarray analysis

One hundred and forty-six (146) expressed sequence tags (ESTs) were up-regulated (Supplementary Table 1) and 429 ESTs were down-regulated (Supplementary Table 2) in response to dietary supplementation with $0.8 \%$ Arg between Days 14 and 25 of gestation. Some of the up-regulated and down-regulated genes with known physiological functions are summarized in Tables 2 and 3, respectively. Among the up-regulated genes in the placentae of Arg-supplemented gilts, the mRNA level of troponin $\mathrm{T}$ type 3 (TNNT3) was the greatest, followed by leucine-rich repeat-containing protein 51-like, calcitonin receptor, presenilin 2, ceroid-lipofuscinosis, and leucinerich repeat-containing protein 18-like in descending order. Among the down-regulated genes in the placentae of Argsupplemented gilts, the reduction in the placental mRNA for cytochrome $b$ was the greatest, followed by Ras GEF 
Table 2. Selected gene expression in the porcine placentae was up-regulated by dietary supplementation with $0.8 \%$ L-arginine between Days 14 and 25 of gestation in comparison with effects of the control diet.

\begin{tabular}{|c|c|c|c|c|}
\hline Expressed sequence tag (EST; gene ID) & Accession No. & Gene name & Fold change & $p$-Value \\
\hline BX918610 & NM_001001863 & Troponin T type 3 (TNNT3) & 4.61 & 0.004 \\
\hline TC292911* & XM_003129590 & Leucine-rich repeat-containing protein 51-like & 4.49 & 0.001 \\
\hline EW039857 & NM_001742 & Calcitonin receptor (CALCR) on chromosome 7 & 3.23 & 0.038 \\
\hline AK231515 & EU287432 & Presenilin 2 (PSEN2) & 2.31 & 0.006 \\
\hline TC278497* & NM_018941 & Ceroid-lipofuscinosis, neuronal 8 & 2.23 & 0.010 \\
\hline TC289044* & XM_001929300 & Sus scrofa leucine-rich repeat-containing protein 18-like & 2.10 & 0.020 \\
\hline PGM1 & NM_001076903 & Phosphoglucomutase 1 (PGM1) & 1.86 & 0.030 \\
\hline TC275071* & NM_000536 & Recombination activating gene $2(R A G-2)$ & 1.76 & 0.006 \\
\hline TC275071* & AB091391 & Recombination activating gene $2(R A G-2)$ & 1.76 & 0.006 \\
\hline TC274023* & NM_001097446 & Apolipoprotein B mRNA editing enzyme, catalytic polypeptide-like $3 F$ (APOBEC3F) & 1.70 & 0.005 \\
\hline BX666795 & XM_001924347 & Similar to solute carrier organic anion transporter family member $3 \mathrm{Al}$ (SLCO3A1) & 1.67 & 0.003 \\
\hline TC278155* & NM_214378 & Rh blood group polypeptide & 1.66 & 0.019 \\
\hline TC267605 & NM_001143721 & 6-Phosphofructo-2-kinase/fructose-2,6-biphosphatase 1 (PFKFB1) & 1.55 & 0.025 \\
\hline EW660666 & NM_001045886 & Phenazine biosynthesis-like protein domain containing & 1.54 & 0.013 \\
\hline TC246855* & AY208121 & Myostatin & 1.54 & 0.022 \\
\hline AY610045 & XM_001924474 & Similar to androgen-induced 1 & 1.42 & 0.018 \\
\hline DY428406 & NG_016762 & Pyruvate dehydrogenase kinase isozyme 3 (PDK3) & 1.40 & 0.042 \\
\hline CF361829 & A9YMB8 & NADH dehydrogenase subunit 2 & 1.34 & 0.012 \\
\hline AJ947745 & NG_007956 & Cytochrome P450 family 20 subfamily A polypeptide 1 (CYP20A1) & 1.33 & 0.022 \\
\hline TC261962* & EW422073 & Hemoglobin subunit epsilon 1 (HBE1) & 1.31 & 0.035 \\
\hline AK234630 & XM_001927389 & FK506 (Tacrolimus)-binding protein & 1.28 & 0.009 \\
\hline AJ584674 & NM_213757 & Beta-Galactoside alpha-2,3-sialyltransferase 4 (ST3GAL4) & 1.27 & 0.000 \\
\hline BW980922 & XM_001113023 & dUTP pyrophosphatase isoform 2 transcript variant 4 (DUT) & 1.27 & 0.021 \\
\hline AK239509 & AB529869 & Peroxisomal trans-2-enoyl-CoA reductase (PECR) & 1.25 & 0.027 \\
\hline BX667232 & XM_001925672 & Similar to pecanex-like protein 1 & 1.23 & 0.030 \\
\hline CN155716 & EU617320 & Small calcium-binding mitochondrial carrier 1 & 1.23 & 0.038 \\
\hline EV880225 & DQ629170 & Ribosomal protein S6 (RPS6) & 1.22 & 0.017 \\
\hline CK467702 & NM_001035277 & Cadherin 13 (CDH13) & 1.22 & 0.013 \\
\hline CD572284 & AJ009912 & Proteolipid protein $(P L P)$ & 1.21 & 0.006 \\
\hline TC258084 & NM_006690 & Matrix metallopeptidase 24 (MMP24) & 1.19 & 0.045 \\
\hline DN125568 & GQ184633 & Cell division cycle 2 (CDC2) & 1.18 & 0.048 \\
\hline EW299999 & XM_001498308 & Similar to eukaryotic translation elongation factor 1 beta 2 (EF1 $\beta 2)$ & 1.17 & 0.015 \\
\hline TC258796 & XM_001928025 & Calcineurin A protein transcript variant 2 & 1.14 & 0.049 \\
\hline SCYE1 & NM_001114283 & Aminoacyl tRNA synthetase complex-interacting multifunctional protein 1 (AIMP1) & 1.12 & 0.026 \\
\hline
\end{tabular}

*Sequence can be accessed on http://compbio.dfci.harvard.edu/cgi-bin/tgi. 
effects of the control diet.

\begin{tabular}{|c|c|c|c|c|}
\hline Expressed sequence tag (EST; gene ID) & Accession No. & Gene name & Fold change & $p$-Value \\
\hline AJ964783 & O48246 & Cytochrome b & 0.15 & 0.001 \\
\hline BI341657 & XM_001926447 & RasGEF domain $1 A$ & 0.18 & 0.013 \\
\hline TC273367* & XM_003129699 & Probable dolichyl pyrophosphate GMGGT-like & 0.20 & 0.010 \\
\hline TC257543* & XM_001927988 & Doublecortin domain-containing protein 2 (RU2S) & 0.23 & 0.015 \\
\hline DN100844 & FJ263680 & Acetyl-coenzyme A carboxylase alpha & 0.27 & 0.003 \\
\hline NP321728 & AF274712 & Pig endogenous retrovirus group Beta 3 polymerase & 0.29 & 0.014 \\
\hline BI360386 & XM_003133904 & Oncostatin-M-specific receptor subunit beta-like & 0.31 & 0.009 \\
\hline TC238637* & NM_214376 & Amphiregulin & 0.31 & 0.045 \\
\hline CF178669 & AJ427478 & Agouti signaling protein & 0.33 & 0.023 \\
\hline CX061534 & XM_003130350 & Torsin-1A-interacting protein 1-like & 0.40 & 0.007 \\
\hline TC301037* & XM_003357826 & Serine/threonine-protein kinase [doublecortin like kinase 1 (DCLK1)]-like & 0.42 & 0.012 \\
\hline TC243513* & NM_213996 & Rh family, B glycoprotein $(R H B G)$ & 0.45 & 0.006 \\
\hline DN106254 & NM_001098597 & Osteocrin (OSTN) & 0.47 & 0.025 \\
\hline AY577905 & NM_001001861 & Chemokine ( $C-X$-C motif) ligand 2 (CXCL2) & 0.49 & 0.013 \\
\hline TC278652* & NM_214003 & Insulin-like growth factor binding protein 2 & 0.49 & 0.002 \\
\hline BP443132 & XM_864245.3 & Cytochrome P450 family 2 subfamily C member 33 (CYP2C33) & 0.50 & 0.037 \\
\hline AY198323 & NM_214257 & Dipeptidyl peptidase 4 (DPP4) & 0.51 & 0.030 \\
\hline TC280345* & XM_003122165 & Golgin A1 & 0.51 & 0.018 \\
\hline TC290654* & NM_001105290 & Bone morphogenetic protein $7(B m p 7)$ & 0.55 & 0.030 \\
\hline CO989438 & XM_001928917 & Potassium large conductance calcium-activated channel, subfamily $M$, beta member 4 & 0.56 & 0.017 \\
\hline DQ836054 & NM_001097442 & Disabled-1(DAB1) & 0.57 & 0.021 \\
\hline TC270858* & AF228059 & Decay-accelerating factor CD55 & 0.58 & 0.026 \\
\hline CV878027 & XM_001926796 & Sterile alpha motif domain containing $4 A$ (SAMD4A) & 0.58 & 0.018 \\
\hline TC290589* & XM_003132094 & Upstream binding protein 1 & 0.58 & 0.005 \\
\hline CA513725 & XM_003129205 & Heat shock $70 k D a$ protein 4-like & 0.58 & 0.016 \\
\hline EV881857 & XM_003132080 & Sodium bicarbonate cotransporter 3-like & 0.59 & 0.009 \\
\hline TC266622* & XM_003127574 & Methylenetetrahydrofolate reductase $(N A D(P) H)$, transcript variant 1 & 0.60 & 0.018 \\
\hline TC286353* & NM_001243919 & Coupling of ubiquitin conjugation to ER degradation (CUE) domain containing 1 & 0.60 & 0.007 \\
\hline TC250322* & NM_001037965 & Inhibitor of DNA binding 2 & 0.61 & 0.007 \\
\hline CN159399 & NM_001128506 & Charged multivesicular body protein $4 b$-like & 0.61 & 0.012 \\
\hline AK230591 & NM_001128488 & Antizyme inhibitor 1 & 0.62 & 0.016 \\
\hline AK234300 & XM_003125957 & RIB43A-like with coiled-coils protein 2-like & 0.63 & 0.005 \\
\hline TC247541* & XM_003134192 & Pericentriolar material 1 & 0.64 & 0.015 \\
\hline CF181641 & XM_003128338 & Dystonin, transcript variant 2 & 0.64 & 0.015 \\
\hline AK233736 & XM_001927836 & Similar to Down syndrome critical region gene 1-like 1 protein & 0.65 & 0.033 \\
\hline DQ866834 & DQ279926 & Retinoid X receptor alpha (RXRalpha) & 0.65 & 0.047 \\
\hline AB271924 & NM_001099924 & Fibroblast growth factor receptor 2 (FGFR2) & 0.68 & 0.019 \\
\hline AY850382 & NM_001011505 & Kruppel-like factor 13 (KLF13) & 0.68 & 0.006 \\
\hline AB116561 & NM_213772 & Interferon alpha and beta receptor subunit 1 (IFNAR1) & 0.69 & 0.012 \\
\hline
\end{tabular}


Table 3. Continued.

\begin{tabular}{|c|c|c|c|c|}
\hline Expressed sequence tag (EST; gene ID) & Accession No. & Gene name & Fold change & $p$-Value \\
\hline TC248589* & NM_001077215 & Regulator of differentiation 1 (RODI) & 0.70 & 0.025 \\
\hline AY610204 & NM_214296 & Rho family GTPase 3 (RND3) & 0.70 & 0.039 \\
\hline BP142559 & XM_001926474 & A-kinase anchoring protein 13 (AKAP13) & 0.70 & 0.016 \\
\hline TC257240* & XM_001925375 & Similar to positive regulatory $(P R)$ domain containing 1 , with $Z N F$ domain transcript variant 2 & 0.71 & 0.042 \\
\hline AY284842 & AY284842 & Glycerol-3-phosphate acyltransferase (GPAT) & 0.71 & 0.016 \\
\hline AK235700 & NM_001078670 & Interferon regulatory factor 9 & 0.71 & 0.024 \\
\hline AK235466 & DQ105589S2 & CDP-Diacylglycerol Synthase 2 (CDS2) & 0.71 & 0.013 \\
\hline EU095967 & NM_001105286 & TNF receptor associated factor $6(T R A F 6)$ & 0.71 & 0.023 \\
\hline BP444119 & NM_214224 & 4-Hydroxyphenylpyruvate dioxygenase (HPD) & 0.72 & 0.007 \\
\hline AY159788 & NM_214266 & 5'-AMP-activated protein kinase catalytic subunit alpha-2 (PRKAA2) & 0.72 & 0.025 \\
\hline AK235681 & NM_213963 & Peroxisome proliferator-activated receptor gamma coactivator 1-alpha (PPARGC-1) & 0.72 & 0.032 \\
\hline AK240475 & XM_001927539 & Similar to general transcription factor IIH & 0.73 & 0.006 \\
\hline BP446317 & NM_001097440 & Bridging Integrator 1 (BIN1) & 0.73 & 0.036 \\
\hline CK461960 & NM_001162401 & lysophosphatidic acid receptor 2 (LPAR2) & 0.73 & 0.048 \\
\hline BI184146 & XM_001927725 & Prostaglandin F2 receptor inhibitor (PTGFRN) & 0.74 & 0.002 \\
\hline CV875504 & XM_001926134 & Similar to chloride channel 3 & 0.74 & 0.040 \\
\hline EU009401 & NM_001098605 & Patatin-like phospholipase domain containing 2 (PNPLA2) & 0.74 & 0.014 \\
\hline TC261381 & NM_213973 & Heat-shock protein 90 (HSP90) & 0.75 & 0.036 \\
\hline AK233668 & NM_213830 & Folate-binding protein $(F B P)$ & 0.75 & 0.029 \\
\hline AY609622 & AY609622 & Similar to small nuclear RNA activating complex & 0.76 & 0.037 \\
\hline TC299692 & NM_001025107 & Homo sapiens adenosine deaminase RNA-specific (ADAR) & 0.76 & 0.046 \\
\hline DY420532 & NM_017902 & Homo sapiens hypoxia inducible factor 1 alpha subunit inhibitor (HIF1AN) & 0.76 & 0.047 \\
\hline AB254406 & NM_001101814 & Nuclear receptor subfamily 1 group H member 3 (NR1H3) & 0.77 & 0.028 \\
\hline DN120475 & XM_001927228 & Tyrosine 3-monooxygenase/tryptophan 5-monooxygenase activation protein & 0.77 & 0.013 \\
\hline AY644721 & NM_001009581 & Peripherial benzodiazepine receptor associated protein (PAP7) & 0.78 & 0.037 \\
\hline AJ955195 & XM_001929149 & Similar to transmembrane protein 77 & 0.79 & 0.036 \\
\hline AK237448 & XM_001928092 & Similar to Rab-1C & 0.79 & 0.033 \\
\hline AK234427 & XM_001928746 & Similar to adenosine deaminase-like protein & 0.79 & 0.046 \\
\hline TC278200* & XM_001925656 & Similar to procollagen & 0.79 & 0.038 \\
\hline AK235686 & XM_001925381 & Similar to insulin-degrading enzyme & 0.80 & 0.016 \\
\hline AK237044 & XM_001499279 & Similar to ubiquitin-conjugating enzyme E2Z & 0.80 & 0.034 \\
\hline AK232486 & NM_001159481 & Pyruvate dehydrogenase kinase isozyme 2 (PDK2) & 0.81 & 0.042 \\
\hline DN100853 & AF339885 & Mannose-6-phosphate/insulin-like growth factor II receptor & 0.81 & 0.038 \\
\hline
\end{tabular}

*Sequence can be accessed on http://compbio.dfci.harvard.edu/cgi-bin.

CDP, cytosine diphosphate; RasGEF, Ras (rat sarcoma protein p21) guanine nucleotide exchange factor; GMGGT, Glc1Man9GlcNAc2 alpha-1,3-glucosyltransferase; RIB43A, ribbon protofilament protein $43 \mathrm{~A}$ (43-kDa protein); TNF, tumor necrosis factor; ZNF, zinc finger. 


\begin{tabular}{lll}
\hline Gene name & Species & Databas
\end{tabular}

5,10-methylenetetrahydrofolate reductase (NADPH)

Homo sapiens

KEGG_PATHWAY

Pathway

Acetyl-coenzyme A carboxylase alpha

Homo sapiens KEGG_PATHWAY

hsa00670:One carbon pool by folate

hsa00680:Methane metabolism

hsa00061:Fatty acid biosynthesis

hsa00620:Pyruvate metabolism

hsa00640:Propanoate metabolism

hsa04910:Insulin signaling pathway

Asparagine-linked glycosylation 8, alpha-1,3-glucosyltransferase ho- Homo sapiens $\quad$ KEGG_PATHWAY hsa00510:N-Glycan biosynthesis molog (S. cerevisiae)

Chemokine (C-X-C motif) ligand 2

Sus scrofa KEGG_PATHWAY

Bos taurus KEGG_PATHWAY

sc04062:Chemokine signaling pathway

Inhibitor of DNA binding 2

Sus scrofa

KEGG_PATHWAY

bta04144:Endocytosis

ssc04350:TGF-beta signaling pathway

Oncostatin M receptor

Homo sapiens KEGG PATHWAY

hsa04060:Cytokine-cytokine receptor interaction

hsa04630:Jak-STAT signaling pathway

Potassium large conductance calcium-activated channel, subfamily $M, \quad$ Sus scrofa $\quad$ KEGG_PATHWAY

ssc04270:Vascular smooth muscle contraction

beta member 4

\begin{tabular}{|c|c|c|c|}
\hline Presenilin 2 & Sus scrofa & KEGG_PATHWAY & $\begin{array}{l}\text { ssc04330:Notch signaling pathway } \\
\text { ssc05010:Alzheimer's disease }\end{array}$ \\
\hline Recombination activating gene 2 & Sus scrofa & KEGG_PATHWAY & ssc05340:Primary immunodeficiency \\
\hline Ribosomal protein S6 (RPS6) & Sus scrofa & KEGG_PATHWAY & Protein synthesis \\
\hline Protein for ubiquitin conjugation & Sus scrofa & KEGG_PATHWAY & Protein degradation \\
\hline Antizyme inhibitor 1 & Sus scrofa & KEGG_PATHWAY & Polyamine synthesis \\
\hline Troponin $T$ & Sus scrofa & KEGG_PATHWAY & Cell growth and development \\
\hline Cadherin 13 & Sus scrofa & KEGG_PATHWAY & Cell-cell adhesion in tissues \\
\hline Organic anion transporter & Sus scrofa & KEGG_PATHWAY & Transport of organic anions \\
\hline CYP20A1 & Sus scrofa & KEGG_PATHWAY & Removal of xenobiotics \\
\hline Heat shock $70 k$ Da protein 4-like & Sus scrofa & KEGG_PATHWAY & Inflammation and oxidative stress \\
\hline Acetyl-coenzyme A carboxylase alpha & Homo sapiens & BIOCARTA & Leptin Pathway:Reversal of Insulin Resistance by Leptin \\
\hline 5,10-Methylenetetrahydrofolate reductase (NADPH) & Homo sapiens & PANTHER_PATHWAY & P02743:Formyltetrahydroformate biosynthesis \\
\hline Doublecortin-like kinase 1 & Homo sapiens & PANTHER_PATHWAY & P00031:Inflammation mediated by chemokine and cytokine signaling pathway \\
\hline 5,10-Methylenetetrahydrofolate reductase (NADPH) & Homo sapiens & REACTOME_PATHWAY & REACT_11193:Metabolism of vitamins and cofactors \\
\hline \multirow{2}{*}{ Acetyl-coenzyme A carboxylase alpha } & \multirow{2}{*}{\multicolumn{2}{|c|}{ Homo sapiens REACTOME_PATHWAY }} & REACT_1505:Integration of energy metabolism \\
\hline & & & REACT_602:Metabolism of lipids and lipoproteins \\
\hline Pericentriolar material 1 & \multicolumn{2}{|c|}{ Homo sapiens REACTOME_PATHWAY } & REACT_152:Cell cycle, mitotic \\
\hline
\end{tabular}

KEGG, a database resource for understanding the metabolic network and functions of the biological system; STAT, signal transducer and activator of transcription; TGF, transforming growth factor. 
domain 1A-similar gene, probable dolichyl pyrophosphate GMGGT-like gene, doublecortin domain-containing protein 2 (RU2S), acetyl-coenzyme A carboxylase alpha, and pig endogenous retrovirus group beta3 polymerase in descending order.

The placental expression of mRNAs for the following enzymes or proteins related to amino acid metabolism did not differ $(p>0.05)$ between the control and $0.8 \%$ Arg-supplemented gilts: arginase I, ornithine carbamoyltransferase, acetylornithine and succinylornithine aminotransferase, pyrroline-5-carboxylate reductase 1, creatine kinase (mitochondrial) 2, guanidinoacetate N-methyltransferase, glutamine synthetase, glutaminase, glutamine:fructose-6-phosphate aminotransferase, carbamoyl-phosphate synthetase I, glutamine-dependent carbamoyl-phosphate synthase, glutamate decarboxylase, $\mathrm{N}$-acetylglutamate synthase, aspartate aminotransferase 1 (glutamic-oxaloacetic transaminase 1, cytosolic), aspartate aminotransferase 2 (glutamic-oxaloacetic transaminase 2, mitochondrial), aspartate kinase, aspartate carbamoyltransferase, argininosuccinate synthetase, the glycine cleavage system $\mathrm{H}$ protein, glycine $\mathrm{N}$-methyltransferase, lysine $\alpha$-ketoglutarate reductase/saccharopine dehydrogenase, L-pipecolic acid oxidase, methionine-R-sulfoxide reductase B3, S-adenosylmethionine decarboxylase, Sadenosylhomocysteine hydrolase, betaine-homocysteine S-methyltransferase, methionyl-tRNA formyltransferase, prolyl 4-hydroxylase $\alpha$ subunit, prolyl 4-hydroxylase isoform c, threonyl-tRNA synthetase, serine racemase, homoserine kinase, kynurenine 3-monooxygenase (kynurenine 3-hydroxylase), mechanistic target of rapamycin (MTOR), selenocysteine-specific translation elongation factor, dopamine beta-hydroxylase, urate oxidase, monoamine oxidase $\mathrm{B}, \mathrm{D}$-amino acid oxidase, glutathione peroxidase 5, glutathione S-transferase, solute carrier family 7 member 1 (SLC7A1 encoding for the CAT1 protein), and the branched-chain amino acid transport system carrier protein.

Dietary supplementation with $0.8 \%$ Arg did not affect $(p>0.05)$ the placental expression of mRNAs for the following enzymes or proteins related to glucose, fructose, fatty acid, and vitamin metabolism, as well as the Krebs cycle, the mitochondrial respiratory chain, hydroxylation, peroxidation, and water transport: glucose transporter 5, lactate dehydrogenases (A and C), hexokinase II, fructose1,6-bisphosphatase, fructose-1,6-bisphosphate aldolase, 6phosphofructo-2-kinase/fructose-2,6-biphosphatase 1, aldolase $\mathrm{B}$, ketohexokinase isoform $\mathrm{A}$, pyruvate dehydrogenase E1 component alpha subunit, dihydrolipoamide Sacetyltransferase (E2 component of pyruvate dehydrogenase complex), pyruvate kinase II, pyruvate dehydrogenase kinase isozymes ( 1 and 4), glucose-6-phosphate dehydrogenase, malate dehydrogenase $1\left(\mathrm{NAD}^{+}\right.$, soluble), malate dehydrogenase $\left(\mathrm{NADP}^{+}\right)$, 25-hydroxyvitamin $\mathrm{D}_{3} 1 \alpha$ hydroxylase, pyridoxine-5'-phosphate oxidase, NADPH oxidase 4, cytochrome oxidase subunits (I, II, and III), cytochrome c oxidases (I and III), methylmalonatesemialdehyde dehydrogenase, medium-chain acyl-CoA dehydrogenase, acyl-coenzyme A oxidase 2, peroxisomal, succinyl-CoA: $\alpha$-ketoacid coenzyme A transferase, citrate synthase, isocitrate dehydrogenase $3\left(\mathrm{NAD}^{+}\right)$beta, isocitrate dehydrogenase $\left(\mathrm{NAD}^{+}\right)$subunit 1 , cytosolic $\mathrm{NADP}^{+}$dependent isocitrate dehydrogenase, succinate dehydrogenase subunits (A, B and D), NADH dehydrogenase (ubiquinone) 1 beta subcomplex, NADH dehydrogenase subunits (1, 3, 4 and 6), cytochrome oxidase subunit I, cytochrome P450 21-hydroxylase, cytochrome P-450 17ahydroxylase, cytidine monophosphate- $\mathrm{N}$-acetylneuraminic acid hydroxylase, peroxidase precursor, and aquaporins (1, $3,4,5,7,8,9,11$, and 12).

\subsection{DAVID analysis of differentially expressed genes in porcine placentae}

The functional analysis by the DAVID program revealed that the genes with altered expression are related to nutrient transport, protein synthesis, protein degradation, polyamine synthesis, ion transport, glucose metabolism, fatty acid biosynthesis, immune development, inflammation, and anti-oxidative responses, as well as insulin, transforming growth factor beta, and Notch signaling pathways (Table 4). Changes in metabolic pathways were associated with alterations in the expression of single genes or a group of related genes.

\subsection{Interaction pathways analysis for selected genes that were differentially expressed in the placentae of Arg-supplemented gilts}

Table 5 summarizes the results of the GO terms and KEGG interaction pathways for selected genes that were differentially expressed in the placentae of argininesupplemented gilts. We noted that supplementing Arg to the diet of gestating gilts influenced the following interaction pathways: phosphoinositide 3-kinase (PI3K)protein kinase $\mathrm{B}$ (Akt) signaling pathway, regulation of circadian rhythm, glucagon signaling pathway, cell surface determinants, inflammation, osteoclast differentiation, Hippo signaling pathway, membranous septum morphogenesis, nitrogen utilization, mesenchymal cell differentiation, branching involved in salivary gland morphogenesis, ammonium transmembrane transport, organic cation transport, mesenchymal cell differentiation, beta-amyloid metabolic process, positive regulation of astrocyte differentiation, nutrient oxidation, extracellular space metabolism and remodeling, cell growth and development, regulation of gene transcription, and embryonic pattern specification.

\subsection{Change in placental mRNA levels based on RT-PCR analysis}

Data from the RT-PCR analysis of selected genes largely confirmed results from the microarray analysis (Table 6). These genes were chemokine (C-X-C motif) 
Table 5. Interaction pathways analysis for selected genes that were differentially expressed in the placentae of arginine-supplemented gilts.

\begin{tabular}{|c|c|c|c|c|}
\hline Term & Count & $\%$ & $p$-Value & Genes \\
\hline ssc04151:PI3K-Akt signaling pathway & 7 & 13.46154 & 0.001015 & $\begin{array}{c}N M_{-} 213973, X M \_001927228, N M \_214266, N M \_001099924 \\
N M \_001162401, N M \_213772, X M \_003133904\end{array}$ \\
\hline GO:0042752 regulation of circadian rhythm & 3 & 5.769231 & 0.004521 & NM_213963, NM_214266, NM_001037965 \\
\hline ssc04922:glucagon signaling pathway & 4 & 7.692308 & 0.005019 & XM_001928025, NM_213963, NM_001143721,NM_214266 \\
\hline GO:0009986 cell surface determinants & 5 & 9.615385 & 0.008898 & $\begin{array}{c}X M \_001925381, N M \_001114283, N M \_214376, N M \_214257 \\
N M \_001162401\end{array}$ \\
\hline ssc05160:inflammation & 4 & 7.692308 & 0.011177 & NM_001101814, NM_001078670,NM_001105286, NM_213772 \\
\hline ssc04380:osteoclast differentiation & 4 & 7.692308 & 0.012663 & XM_001928025, NM_001078670,NM_001105286, NM_213772 \\
\hline ssc04390:Hippo signaling pathway & 4 & 7.692308 & 0.013715 & NM_001105290,XM_001927228,NM_214376, NM_001037965 \\
\hline GO:0003149 membranous septum morphogenesis & 2 & 3.846154 & 0.01435 & NM_001099924, NM_001037965 \\
\hline GO:0019740 nitrogen utilization & 2 & 3.846154 & 0.01435 & NM_213996, NM_214378 \\
\hline GO:0060445 branching involved in salivary gland morphogenesis & 2 & 3.846154 & 0.017907 & NM_001105290,NM_001099924 \\
\hline GO:0072488 ammonium transmembrane transport & 2 & 3.846154 & 0.02145 & NM_213996, NM_214378 \\
\hline GO:0015695 organic cation transport & 3 & 3.846154 & 0.024981 & $N M \_213996, N M \_214378, N M \_214376$ \\
\hline GO:0048762 mesenchymal cell differentiation & 2 & 3.846154 & 0.024981 & NM_001105290, NM_001099924 \\
\hline GO:0050435 beta-amyloid metabolic process & 2 & 3.846154 & 0.0285 & XM_001925381, NM_001078666 \\
\hline GO:0048711 positive regulation of astrocyte differentiation & 2 & 3.846154 & 0.0285 & NM_001097440,NM_001037965 \\
\hline GO:0014850 nutrient oxidation & 2 & 3.846154 & 0.0285 & $N M \_213963, N M \_214266$ \\
\hline GO:0005615 extracellular space metabolism and remodeling & 7 & 13.46154 & 0.032712 & XM_001925381,NM_001105290,NM_001114283, NM_001098597, \\
\hline- & - & - & - & NM_214376, NM_001001861, NM_214003 \\
\hline GO:0060749 cell growth and development & 2 & 3.846154 & 0.038982 & NM_214376, NM_001037965 \\
\hline GO:0048557 regulation of gene transcription & 2 & 3.846154 & 0.042451 & NM_001099924,NM_001037965 \\
\hline GO:0009880 embryonic pattern specification & 2 & 3.846154 & 0.042451 & NM_001105290,NM_001099924 \\
\hline
\end{tabular}

PI3K, phosphoinositide 3-kinase. 
Table 6. Verification of microarray analysis data using quantitative RT-PCR analyses.

\begin{tabular}{lccccc}
\hline \multirow{2}{*}{ Accession No. } & \multirow{2}{*}{ Gene name } & \multicolumn{2}{c}{ Microarray analysis } & \multicolumn{2}{c}{ RT-PCR analysis } \\
\cline { 3 - 6 } & & Fold change & $p$-Value & Fold change & $p$-Value \\
\hline NM_001001863 & TNNT3 & 4.61 & 0.004 & 4.37 & 0.015 \\
AK231515 & Presenilin 2 & 2.31 & 0.006 & 1.77 & 0.021 \\
TC275071 & RAG-2 & 1.76 & 0.006 & 1.68 & 0.013 \\
TC267605 & PFKFB1 & 1.55 & 0.025 & 1.51 & 0.036 \\
XM_001926447 & RasGEF & 0.18 & 0.013 & 0.22 & 0.019 \\
NM_001001861 & CXCL2 & 0.49 & 0.013 & 0.43 & 0.016 \\
TC243513 & RHBG & 0.45 & 0.006 & 0.63 & 0.016 \\
TC257543 & RU2S & 0.23 & 0.015 & 0.38 & 0.044 \\
EU288086 & MTOR & 1.01 & 0.783 & 1.04 & 0.520 \\
NM_001012613 & SLC7A1 & 0.97 & 0.602 & 0.94 & 0.550
\end{tabular}

CXCL2, chemokine (C-X-C motif) ligand 2; MTOR, mechanistic target of rapamycin; PFKFB1, 6-Phosphofructo-2-kinase/fructose-2,6-biphosphatase 1; RAG-2, recombination activating gene 2; RasGEF, Ras (rat sarcoma protein p21) guanine nucleotide exchange factor; RHBG, Rh family, B glycoprotein; RU2S, doublecortin domaincontaining protein 2; SLC7A1, Sus scrofa solute carrier family 7 member 1 (CAT-1); TNNT3, troponin T type 3.

ligand 2 (CXCL2), MTOR, presenilin 2, 6-phosphofructo2-kinase/fructose-2,6-biphosphatase 1 (PFKFB1), recombination activating gene 2 (RAG-2), Ras (rat sarcoma protein p21) guanine nucleotide exchange factor (RasGEF), Rh family B glycoprotein (RHBG), RU2S, SLC7A1, and TNNT3.

\section{Discussion}

The placenta plays a critical role in transporting amino acids from mother to fetus, thereby having an enormous impact on fetal survival, growth, and development [18]. The pig has true epitheliochorial placentation, meaning that the placenta is only superficially attached to the uterine luminal epithelium. Such a placental structure increases the efficiency of gas and nutrient exchanges between fetus and mother [19]. Consistent with the increased availability of Arg in the conceptus of Arg-supplemented gilts [4], results of this microarray analysis revealed that dietary supplementation with $0.8 \%$ Arg to gilts between Days 14 and 25 of gestation altered the expression of 575 genes in their placentae. To our knowledge, this is the first study of effects of dietary Arg supplementation on in vivo expression of placental genes in any animal species. The microarray assay provides a powerful molecular technology to allow for the simultaneous determination of the expression of thousands of genes (particularly unexpected ones) in a tissue. The results can facilitate the elucidation of mechanisms responsible for the effects of nutrients or other substances.

Polyamines are crucial for cell growth, migration, and proliferation, as well as angiogenesis [20]. We recently reported that dietary supplementation with Arg to gilts increased the activity of ornithine decarboxylase (ODC) and the synthesis of polyamines from ornithine in their placenta [4]. A novel and unexpected finding of the present study is that Arg supplementation reduced the placental expression of ODC antizyme inhibitor 1 (Table 3). This inhibitor protein binds to and destabilizes ODC, thereby suppressing ODC activity. Thus, a decrease in the expression of the ODC antizyme inhibitor 1 alleviates the inhibitory effect on ODC activity, leading to enhanced polyamine synthesis in placentae. This action of Arg is associated with an increase in the transmembrane transport of $\mathrm{Ca}^{2+}$ in the porcine placentae (Table 2), which further stimulates ODC activity in mammalian cells [21].

Results of our previous in vitro studies revealed that, as compared with $10 \mu \mathrm{M} \mathrm{Arg}$, augmentation of Arg concentration in culture media from 50 to $350 \mu \mathrm{M}$ dosedependently increased protein synthesis and inhibited protein degradation as well as the proliferation of trophectoderm cells partly via a mechanism requiring MTOR activation [9]. Leucine and glutamine also activate the MTOR cell signaling pathways in placental cells and embryos [2225]. Other underlying mechanisms likely require the following six regulatory pathways. The first pathway is related to increases in the placental expression of aminoacyl tRNA synthetase complex-interacting multifunctional protein 1, ribosomal protein S6 (a component of the 40S ribosomal subunit for mRNA translation), eukaryotic translation elongation factor 1 beta 2 , and cell division cycle 2 (Table 2), leading to increased protein synthesis. The second pathway may require an increase in the placental expression of dUTP pyrophosphatase (Table 2), which is critical for the fidelity of DNA replication and repair [26]. The third pathway involves decreases in the placental expression of ubiquitin- 
conjugating proteins, resulting in a reduction in intracellular proteolysis. Fourth, an increase in the expression of type-3 troponin may beneficially enhance the growth of the placenta and alter its structure, as reported for myogenesis [27], to allow for the efficient transfer of nutrients and oxygen from mother to fetus [5]. Fifth, the up-regulated expression of leucine-rich repeat-containing proteins in the placenta of Arg-supplemented gilts may facilitate gene transcription, as reported for other cell types [28] to enhance the receptivity of the organs to Arg or its metabolites in placental cells [29]. Sixth, in coordination with all these changes, downregulated expression of insulin-like growth factor 2 (IGF-2) binding protein can enhance the availability of IGF-2 to promote placental cell growth and differentiation via phosphoinositide 3 (PI3) and mitogen-activated protein (MAP) kinase signaling pathways [30]. Thus, collectively, Arg regulates intracellular protein turnover to favor protein accretion in cells and their growth through multiple mechanisms.

Dietary Arg supplementation enhances placental angiogenesis (the growth of new blood vessels from the existing vasculature) partly via the generation of polyamines and NO [4,5]. In addition, there is emerging evidence that glycans are novel activators of angiogenesis under physiological conditions due to changes in protein glycosylation [31]. Consistent with this notion, the expression of beta-galactoside alpha 2-3 sialyltransferase (a glycosyltransferase), a key enzyme that catalyzes protein glycosylation via the terminal sialylation of glycoproteins and glycolipids, was enhanced in the placentae of Arg-supplemented gilts as compared to the control group (Table 2). Likewise, calcitonin stimulated all phases of angiogenesis through the calcitonin receptor [32], and matrix metallopeptidases contributes to angiogenesis through the degradation of the vascular basement membrane and remodeling of the extracellular matrix [33]. Furthermore, calcineurin (a calcium- and calmodulin-dependent serine/threonine protein phosphatase) stimulates angiogenesis through $\mathrm{Ca}^{2+}$ and calmodulin signaling (including the synthesis of $\mathrm{NO}$ by endothelial NO synthase) in cells [34], whereas presenilin 2 helps to process intracellular proteins that transmit chemical signals (e.g., vascular endothelial growth factor) from the cell membrane into the nucleus [35]. In this regard, it is noteworthy that maternal Arg supplementation augmented the expression of calcitonin receptor, matrix metallopeptidase 24, calcineurin A, and presenilin 2 in porcine placentae (Table 2). Thus, Arg-induced angiogenesis in porcine placentae is supported by multiple mechanisms (Table 5).

Arg is known to alleviate inflammation $[11,36]$ and enhance immune responses [37] in animals but the underlying mechanisms are not fully understood. For example, dietary supplementation with Arg reduces risk for gastrointestinal infections and embryonic deaths in gestating gilts [38]. Interestingly, unexpected results of the present work revealed increases in the placental expression of the following key genes related to anti-oxidative and im- mune responses in gestating gilts supplemented with Arg (Table 3). These genes include: (a) the recombinationactivating genes (RAGs), which encode part of a protein complex that plays important roles in the rearrangement and recombination of the genes for B-cell development and the production of immunoglobulins [39], as well as T-cell receptor molecules [40]; (b) leucine-rich repeat-containing proteins 51-like and 18-like, which promote the maturation of cells of the innate immune system [41,42]; and (c) solute carrier organic anion transporter family member $3 \mathrm{~A} 1$ (SLCO3A1), which encodes for a membrane protein in immune cells that mediates inflammatory processes in epithelial cells through the activation of the NF- $k \mathrm{~B}$ cell signaling pathway [43]. Likewise, dietary supplementation with Arg to gestating gilts reduced the placental expression of mRNAs for heat shock protein 70, hypoxia inducible factor 1 alpha subunit inhibitor, decay-accelerating factor CD55 (that is involved in epithelial inflammation) [44], amphiregulin (a transmembrane glycoprotein that participates in cell inflammatory responses [45]), and CXCL2 (Table 3), indicating an improvement in cellular redox balance and a reduction in cellular inflammation.

There is much evidence that Arg regulates the metabolism of lipids and glucose in mammalian liver, skeletal muscle, and white adipose tissue $[8,46]$, as well as nutrient transport by the small intestine [47]. However, little is known about the roles of Arg in these biochemical processes in placentae. Results of the microarray analysis indicated, for the first time, that dietary supplementation with Arg altered the expression of some key genes in porcine placentae that are involved in: (a) glycolysis and glucose oxidation to $\mathrm{CO}_{2}$; (b) fatty acid synthesis and oxidation; (c) one-carbon unit metabolism; and (d) ion transport (Tables 2 and 3). The up-regulated genes include phosphoglucomutase, PFKFB1, pyruvate dehydrogenase kinase isozyme 3, NADH dehydrogenase subunit 2, peroxisomal trans-2-enoyl-CoA reductase, cytochrome P450 family 20 subfamily A polypeptide 1, apolipoprotein B mRNA editing enzyme, hemoglobin subunit epsilon 1 (for iron-storage), small calcium-binding mitochondrial carrier 1, and solute carrier organic anion transporter family member 3A1. The down-regulated genes include adenosine deaminase, tyrosine 3-monooxygenase/tryptophan 5monooxygenase activation protein, pyruvate dehydrogenase kinase isozyme 2, acetyl-coenzyme A carboxylase alpha, glycerol-3-phosphate acyltransferase, cytosine diphosphate diacylglycerol synthase 2 , sodium bicarbonate cotransporter 3-like, chloride channel 3, potassium large conductance calcium-activated channel subfamily $\mathrm{M}$, and methylenetetrahydrofolate reductase. The changes in the expression of the metabolic enzymes were associated with those for cell signaling protein, including FK506 (Tacrolimus)-binding protein, retinoid $\mathrm{X}$ receptor alpha, Kruppel-like factor 13 (zinc finger transcription factor), insulin-degrading enzyme, Rho family GTPase 3, inter- 
feron alpha and beta receptor subunit 1, general transcription factor IIH, hypoxia inducible factor 1 alpha subunit inhibitor, and mannose-6-phosphate/insulin-like growth factor II receptor (Tables 2 and 3). Future metabolic studies involving isotope tracers are required to determine actual changes in the rates of placental nutrient transfer, synthesis, and catabolism in Arg-supplemented dams.

Another novel and important finding from the current work is that dietary Arg supplementation increased cadherin expression in porcine placentae (Table 2). Cadherin is a transmembrane protein that mediates cell-cell adhesion [48]. By regulating the stability of contacts between cells, cadherins play a crucial role in tissue morphogenesis and homeostasis. This is consistent with the analysis of interaction pathways for differentially expressed genes (Table 5) and the report that the apparent adhesion force between the chorioallantoic membrane and the endometrial epithelium was greater in Arg-supplemented gilts than control gilts [49]. Further analysis of the adhesion strength would require mechanical testing equipment.

\section{Conclusions}

Dietary supplementation with $0.8 \%$ Arg to gilts between Days 14 and 25 of gestation increased the expression of mRNAs for the syntheses of polyamines and protein, angiogenesis, cell-to-cell interactions, immune development, and antioxidative responses in placentae. Arginine supplementation reduced the placental expression of genes for protein degradation, inflammation, and cell injury. Furthermore, some of the key genes for glucose and fatty acid metabolism, ion transport, and cell signaling in placentae were differentially expressed between control and Arg-supplemented gilts to support placental growth and differentiation. Results from this microarray study will help to elucidate complex mechanisms responsible for the beneficial effects of Arg in improving conceptus growth, survival, and development in swine and possibly other mammals.

\section{Author contributions}

GW, FWB, and GAJ conceived and designed the study. XL, GW, FWB, GAJ, and RCB performed the experiment. XL and HZ analyzed the data. XL and GW summarized the results and wrote the manuscript. All authors contributed to data interpretation and manuscript revisions, and approved the final manuscript.

\section{Ethics approval and consent to participate}

This study was approved by The Institutional Animal Care and Use Committee of Texas A\&M University. No consent to participate was applicable.

\section{Acknowledgment}

Not applicable.

\section{Funding}

This work was supported by Agriculture and Food Research Initiative Competitive Grants (2015-67015-23276) from the USDA National Institute of Food and Agriculture.

\section{Conflict of interest}

The authors declare no conflict of interest. $\mathrm{GW}$ is serving as one of the Editorial Board members of this journal. We declare that GW had no involvement in the peer review of this article and has no access to information regarding its peer review. Full responsibility for the editorial process for this article was delegated to GP.

\section{Supplementary material}

Supplementary material associated with this article can be found, in the online version, at https://www.imrpre ss.com/journal/FBL/27/1/10.31083/j.fbl2701033.

\section{References}

[1] Gilbreath KR, Bazer FW, Satterfield MC, Wu G. Amino acid nutrition and reproductive performance in ruminants. Advances in Experimental Medicine and Biology. 2021; 1285: 43-61.

[2] Wu G, Bazer FW, Johnson GA, Hou YQ. Arginine nutrition and metabolism in growing, gestating and lactating swine. Journal of Animal Science. 2018; 96: 5035-5051.

[3] Zhang Q, Hou YQ, Bazer FW, He WL, Posey EA, Wu G. Amino acids in swine nutrition and production. Advances in Experimental Medicine and Biology. 2021; 1285: 81-107.

[4] Elmetwally MA, Li XL, Johnson GA, Burghardt RC, Herring $\mathrm{CM}$, Kramer $\mathrm{AC}$, et al. Dietary supplementation with Larginine between Days 14 and 25 of gestation enhances NO and polyamine syntheses and expression of angiogenic proteins in porcine placentae. Amino Acids. 2021. (in press)

[5] Wu G, Bazer FW, Johnson GA, Herring C, Seo H, Dai ZL, et al. Functional amino acids in the development of the pig placenta. Molecular Reproduction and Development. 2017; 84: 879-882.

[6] Zhu C, Li XL, Bazer FW, Johnson GA, Burghardt RC, Jiang ZY, et al. Dietary L-arginine supplementation during days 14-25 of gestation enhances aquaporin expression in the placentae and endometria of gestating gilts. Amino Acids. 2021; 53: 1287 1295 .

[7] Wu G. Amino acids: metabolism, functions, and nutrition. Amino Acids. 2009; 37: 1-17.

[8] Wu G. Amino Acids: Biochemistry and Nutrition. 2nd edition. CRC Press: Boca Raton, Florida. 2022.

[9] Kong XF, Tan BE, Yin YL, Gao HJ, Li XL, Jaeger LA, et al. L-Arginine stimulates the mTOR signaling pathway and protein synthesis in porcine trophectoderm cells. Journal of Nutritional Biochemistry. 2012; 23: 1178-1183.

[10] Kong XF, Wang XQ, Yin YL, Li XL, Gao HJ, Bazer FW, et al. Putrescine stimulates the mTOR signaling pathway and protein synthesis in porcine trophectoderm cells. Biology of Reproduction. 2014; 91: 106.

[11] Jobgen W, Fu WJ, Gao H, Li P, Meininger CJ, Smith SB, et al. High fat feeding and dietary L-arginine supplementation differentially regulate gene expression in rat white adipose tissue. Amino Acids. 2009; 37: 187-198.

[12] Li XL, Bazer FW, Johnson GA, Burghardt RC, Frank JW, Dai ZL, et al. Dietary supplementation with L-arginine between days 14 and 25 of gestation enhances embryonic development and survival in gilts. Amino Acids. 2014; 46: 375-384. 
[13] Chiang HI, Swaggerty CL, Kogut MH, Dowd SE, Li X, Pevzner IY, et al. Gene expression profiling in chicken heterophils with Salmonella enteritidis stimulation using a chicken $44 \mathrm{~K}$ Agilent microarray. BMC Genomics. 2008; 9: 526.

[14] Dennis G Jr, Sherman BT, Hosack DA, Yang J, Gao W, Lane HC, et al. DAVID: database for annotation, visualization, and integrated discovery. Genome Biology. 2003; 4: R60.

[15] Huang DW, Sherman BT, Lempicki RA. Systematic and integrative analysis of large gene lists using DAVID Bioinformatics Resources. Nature Protocols. 2009; 4: 44-57.

[16] Huang DW, Sherman BT, Lempicki RA. Bioinformatics enrichment tools: paths toward the comprehensive functional analysis of large gene lists. Nucleic Acids Research. 2009; 37: 1-13.

[17] Fu WJ, Stromberg AJ, Viele K, Carroll RJ, Wu G. Statistics and bioinformatics in nutritional sciences: analysis of complex data in the era of systems biology. Journal of Nutritional Biochemistry. $2010 ; 21$ : $561-572$.

[18] Reynolds LP, McLean KJ, McCarthy KL, Diniz WJS, Menezes ACB, Forcherio JC, et al. Nutritional regulation of embryonic survival, growth and development. Advances in Experimental Medicine and Biology. 2022; 1354: 63-76.

[19] Johnson GA, Bazer FW, Seo H. The early stages of implantation and placentation in the pig. Advances in Anatomy, Embryology, and Cell Biology. 2021; 234: 61-89.

[20] Halloran KM, Stenhouse C, Wu G, Bazer FW. Arginine, agmatine and polyamines: Key regulators of conceptus development in mammals. Advances in Experimental Medicine and Biology. 2021; 1332: 85-105.

[21] Langdon RC, Fleckman P, McGuire J. Calcium stimulates ornithine decarboxylase activity in cultured mammalian epithelial cells. Journal of Cellular Physiology. 1984; 118: 39-44.

[22] Carrera AC. TOR signaling in mammals. Journal of Cell Science. 2004; 117: 4615-4616.

[23] Kim JY, Burghardt RC, Wu G, Johnson GA, Spencer TE, Bazer FW. Select Nutrients in the Ovine Uterine Lumen. VIII. Arginine stimulates proliferation of ovine trophectoderm cells through MTOR-RPS6K-RPS6 signaling cascade and synthesis of nitric oxide and polyamines. Biology of Reproduction. 2011; 84: 70-78

[24] Martin PM, Sutherland AE. Exogenous amino acids regulate trophectoderm differentiation in the mouse blastocyst through an mTOR-dependent pathway. Developmental Biology. 2001; 240: 182-193.

[25] Wullschleger S, Loewith R, Hall MN. TOR signaling in growth and metabolism. Cell. 2006; 124: 471-484.

[26] Gadsden MH, McIntosh EM, Game JC, Wilson PJ, Haynes RH. dUTP pyrophosphatase is an essential enzyme in Saccharomyces cerevisiae. EMBO Journal. 1993; 12: 4425-4431.

[27] Wong TS, Ordahl CP. Troponin T gene switching is developmentally regulated by plasma-borne factors in parabiotic chicks. Developmental Biology. 1996; 180: 722-744.

[28] Warfel NA, Niederst M, Stevens MW, Brennan PM, Frame MC, Newton AC. Mislocalization of the E3 ligase, beta-transducin repeat-containing protein 1 , in glioblastoma uncouples negative feedback between the pleckstrin homology domain leucinerich repeat protein phosphatase 1 and Akt. Journal of Biological Chemistry. 2011; 286: 19777-19788.

[29] Bazer FW, Johnson GA, Wu G. Amino acids and conceptus development during the peri-implantation period of pregnancy. Advances in Experimental Medicine and Biology. 2015; 843: 23-52.

[30] Kim JY, Song GH, Gao HJ, Farmer JL, Satterfield MC, Burghardt RC, et al. Insulin-like growth factor 2 (IGF2) activates PI3K-AKT1 and MAPK cell signaling pathways and stimulates migration of ovine trophectoderm cells. Endocrinology.
2008; 149: 3085-3094.

[31] Zhong C, Li P, Argade S, Liu L, Chilla A, Liang W, et al. Inhibition of protein glycosylation is a novel pro-angiogenic strategy that acts via activation of stress pathways. Nature Communications. 2020; 11: 6330 .

[32] Chigurupati S, Kulkarni T, Thomas S, Shah G. Calcitonin stimulates multiple stages of angiogenesis by directly acting on endothelial cells. Cancer Research. 65: 8519-8529.

[33] Rundhaug JE. Matrix metalloproteinases and angiogenesis. Journal of Cellular and Molecular Medicine. 2005; 9: 267-285.

[34] Siamakpour-Reihani S,Caster J,Nepal DB,Courtwright A,Hilliard E,Usary $\mathrm{J}$, et al. The role of calcineurin/NFAT in SFRP2 induced angiogenesis-A rationale for breast cancer treatment with the calcineurin inhibitor tacrolimus. PLoS ONE. 2011; 6: e20412.

[35] Thinakaran G, Parent AT. Identification of the role of presenilins beyond Alzheimer's disease. Pharmacological Research. 2004; 50: 411-418.

[36] Tan BE, Yin YL, Kong XF, Li P, Li XL, Gao HJ, et al. L-Arginine stimulates proliferation and prevents endotoxin-induced death of intestinal cells. Amino Acids. 2010; 38: 1227-1235.

[37] Li P, Wu G. Important roles of amino acids in immune responses. British Journal of Nutrition. 2021. (in press)

[38] Kim SW, Mateo RD, Yin YL, Wu G. Functional amino acids and fatty acids for enhancing production performance of sows and piglets. Asian-Australasian Journal of Animal Science. 2007; 20: $295-306$

[39] Ohmori H, Hikida M. Expression and function of recombination activating genes in mature B cells. Critical Reviews in Immunology. 1998; 18:221-235.

[40] McMahan CJ, Fink PJ. RAG reexpression and DNA recombination at $\mathrm{T}$ cell receptor loci in peripheral $\mathrm{CD} 4+\mathrm{T}$ cells. Immunity. 1998; 9: 637- 647.

[41] Eitas TK, Chou W-C, Wen H, Gris D, Robbins GR, Brickey $\mathrm{J}$, et al. The nucleotide-binding leucine-rich repeat (NLR) family member NLRX1 mediates protection against experimental autoimmune encephalomyelitis and represses macrophage/microglia-induced inflammation. Journal of Biological Chemistry. 2014; 289: 4173-4179.

[42] Ng ACY, Eisenberg JM, Heatha RJW, Huetta A, Robinson CM, Nau GJ, et al. Human leucine-rich repeat proteins: a genomewide bioinformatic categorization and functional analysis in innate immunity. Proceedings of the National Academy of Sciences of the United States of America. 2010; 108: 4631-4638.

[43] Lin L, Yee SW, Kim RB, Giacomini KM. SLC transporters as therapeutic targets: emerging opportunities. Nature Reviews Drug Discovery. 2015; 14: 543-560.

[44] Lin F, Spencer D, Hatala DA, Levine AD, Medof ME. Decayaccelerating factor deficiency increases susceptibility to dextran sulfate sodium-induced colitis: Role for complement in inflammatory bowel disease. Journal of Immunology. 2004, 172: 3836-3841.

[45] Zaiss DMW, Gause WC, Osborne LC, Artis G. Emerging functions of amphiregulin in orchestrating immunity, inflammation, and tissue repair. Immunity. 2015; 42: 216-226.

[46] Jobgen WS, Fried SK, Fu WJ, Meininger CJ, Wu G. Regulatory role for the arginine-nitric oxide pathway in metabolism of energy substrates. Journal of Nutritional Biochemistry. 2006; 17: 571-588.

[47] Wu G. Amino acids in nutrition, health, and disease. Frontiers in Bioscience-Landmark. 2021; 26: 1386-1392.

[48] Maître J-L, Heisenberg C-P. Three functions of cadherins in cell adhesion. Current Biology. 2013; 23: R626-R633.

[49] Li XL. Regulation of porcine conceptus survival and growth by L-arginine. Ph.D. Dissertation. Texas A\&M University, College Station: Texas, USA. 2011. 